\title{
Caracterización del indumento de nueve especies de Loasaceae de Venezuela
}

\section{Indument characterization of nine Loasaceae species from Venezuela}

\author{
Eliana Noguera-Savelli ${ }^{1}$, Damelis Jáuregui ${ }^{2 *}$ y Thirza Ruiz-Zapata ${ }^{2}$ \\ ${ }^{1}$ Fundación Instituto Botánico de Venezuela “Dr. Tobias Lasser”. Avenida Salvador Allende, Plaza Venezuela, Apartado 2156, Caracas 1010-A, \\ Distrito Capital, Venezuela. \\ ${ }^{2}$ Instituto de Botánica Agrícola, Facultad de Agronomía, Universidad Central de Venezuela. Apartado postal 4579, Maracay 2101, Estado Aragua, \\ Venezuela. \\ *Correspondencia: jaureguid@agr.ucv.ve
}

\begin{abstract}
Resumen. En este trabajo se caracterizó el indumento observado en diferentes órganos de 9 especies de Loasaceae presentes en Venezuela, precisando su ubicación en la planta a fin de establecer una posible importancia taxonómica. Este estudio se efectuó tanto al microscopio óptico como al microscopio electrónico de barrido. El indumento está constituido por 2 clases de apéndices epidérmicos: tricomas y emergencias. Los tricomas se clasificaron en tricomas eglandulares uniy multicelulares y tricomas glandulares multicelulares. Los tricomas cónicos y gloquidiados uncinados son comunes en todas las especies estudiadas. Nasa resultó el género con la mayor diversidad de tricomas. Se registra por primera vez la presencia de tricomas dendríticos, exclusivamente en Nasa venezuelensis. Las hojas y ovarios son los órganos que mayor variedad de apéndices mostraron. Gronovia scandens fue la única especie con emergencias, restringidas a los tallos. La morfología del indumento es un carácter de valor diagnóstico a nivel de subfamilia, lo cual se evidencia en la clave.
\end{abstract}

Palabras clave: emergencias, Gronovia, Klaprothia, Mentzelia, Nasa, tricomas.

\begin{abstract}
In this work the epidermal indumentum observed in different organs of 9 species of Loasaceae from Venezuela were characterized, emphasizing their type and location on the plant to establish their possible taxonomic significance. The indumentum was studied using optical and scanning electron microscopy. Two classes of indumentum were observed: trichomes and emergences. The trichomes were classified as uni- and multicellular, non-glandular trichomes, and multicellular glandular trichomes. The conical and uncinate glochidiate trichomes were common in all the species studied. Nasa was the genus with the greatest trichomes diversity. The occurrence of dendritic trichomes is reported for the first time, exclusively in Nasa venezuelensis. Leaves and ovaries are the organs with the greatest variety of appendices. Gronovia scandens is the only species with emergences, which are restricted to the stems. The morphology of the epidermal appendices is a character with diagnostic value at the subfamily level, which is demonstrated in the key.
\end{abstract}

Key words: emergences, Gronovia, Klaprothia, Mentzelia, Nasa, trichomes.

\section{Introducción}

Loasaceae se distingue de otras familias relacionadas por presentar mayormente plantas herbáceas con tricomas multicelulares, por lo general urticantes, variados tipos de estaminodios, pétalos con frecuencia cóncavos, y cápsulas valvadas y espiraladas (Lawrence, 1989). En la actualidad, Loasaceae está incluida en el orden Cornales de la subclase Asteridae (Bremer et al., 1998) debido a la presencia de iridoides. Por otro lado, Judd et al. (2008), coinciden en incluir Loasaceae en el orden monofilético Cornales del

Recibido: 03 octubre 2007; aceptado: 26 junio 2009 clado Asterides por caracteres de secuencias de ADN y morfológicos.

De acuerdo con la clasificación propuesta por Weigend (1997), Loasaceae está conformada por 4 subfamilias: Gronovioideae, Loasoideae, Mentzelioideae y Petalonychoideae. Loasaceae incluye aproximadamente 325 especies en 20 géneros distribuidos a lo largo de América, el género Kissenia con 2 especies en el suroeste de África y sur de Arabia, y el género Plakothira con 3 especies en las islas Marquesas de Polinesia (Weigend, 2003). Las Mentzelioideae y las Loasoideae se caracterizan por presentar 10-200 estambres, pétalos con nervios principales múltiples y frutos con más de una semilla 
(en general muchas), comúnmente dehiscentes. Las Gronovioideae tienen sólo 5 estambres, pétalos planos con un solo nervio principal y fruto indehiscente con una sola semilla (Weigend, 2001).

Estudios moleculares recientes han permitido establecer las relaciones filogenéticas internas de las Loasaceae (Moody et al., 2001; Hufford et al., 2003), revelando la monofilia de las subfamilias Gronovioideae y Loasoideae. Sin embargo, la subfamilia Mentzelioideae es parafilética y requiere de revisión. Por lo tanto, la consideración del análisis de datos morfológicos y anatómicos junto con datos moleculares podría facilitar la resolución de las relaciones internas en la familia. Asimismo, la información anatómica de los tricomas puede ser importante en un análisis que incluya la mayor cantidad de evidencia.

Los estudios del indumento en las Loasaceae son pocos, la mayor parte han estado orientados hacia la morfología floral (Thompson y Ernst, 1967; Hufford, 1988, 1989; Moody y Hufford, 2000). Según Theobald et al. (1979), en diversos grupos de plantas se ha comprobado el valor taxonómico de los tricomas, a pesar de que sus caracteres, como densidad y longitud, reflejan variación condicionada por el ambiente y edad de la planta y raramente pueden usarse para diferenciaciones taxonómicas (Metcalfe y Chalk, 1950; Dostert y Weigend, 1999). Entre las características morfológicas de la familia Loasaceae destacan sus tricomas, los cuales le han dado fama de plantas urticantes, y se han convertido en uno de los caracteres de importancia taxonómica, en la medida en que se ha ido profundizando en el estudio de los miembros de esta familia. Para muchas familias la pubescencia o indumento podría parecer un carácter poco confiable; sin embargo, en el caso de Loasaceae, éste parece ser diagnóstico (Barthlott, 1981; Behnke y Barthlott, 1983). El primer trabajo sobre esta familia donde los tricomas se consideran útiles para la diferenciación específica fue el realizado por Darlington (1934) para el género Mentzelia L. Más tarde, Metcalfe y Chalk (1950) presentan una visión general sobre caracteres morfoanatómicos resaltantes en el tallo y la hoja, con énfasis en los tricomas. Poston y Nowicke (1993) señalaron la existencia de tricomas característicos comunes para las subfamilias Gronovioideae, Loasoideae y Mentzelioideae, los cuales tienen púas rectas y/o púas retrorsas y presentan un ápice agudo a capitado. El tricoma con púas retrorsas o con ganchos curvos hacia abajo o hacia arriba es llamado gloquidiado. Para la subfamilia Gronovioideae, autores como Davis y Thompson (1967) y Poston y Nowicke (1993) coincidieron en señalar para el género Gronovia L. la presencia de un tricoma característico con el ápice en forma de doble gancho, que sólo se presenta en tallos y pecíolos. Para el género Fuertesia Urb. mencionaron la presencia de un tricoma de doble gancho, como el de Gronovia L., y otro apoyado sobre la epidermis con púas retrorsas y 2 brazos de diferentes tamaños. En el género Cevallia Lag., registraron la existencia de tricomas dendríticos. Dostert y Weigend (1999) señalaron que es importante comparar el indumento de diferentes órganos en Loasaceae.

Como parte de la revisión taxonómica de Loasaceae para Venezuela (Noguera, 2006) se caracterizaron los tricomas de los diferentes órganos de las 9 especies encontradas en el país, pertenecientes a 3 de las 4 subfamilias que la conforman de acuerdo con Weigend (1997): Gronovia scandens L. (Gronovioideae); Klaprothia fasciculata (C. Presl) Poston, K. mentzelioides Kunth, Nasa lindeniana (Urb. et Gilg) Weigend, N. perijensis (Weigend) Weigend, N. triphylla Juss. subsp. papaverifolia (Kunth) Weigend, $N$. venezuelensis (Weigend) Weigend (Loasoideae); Mentzelia aspera L., M. scabra Kunth subsp. chilenis (Gay) Weigend (Mentzelioideae) con el objetivo de contribuir al conocimiento de los apéndices epidérmicos presentes en dicho grupo y establecer su posible importancia taxonómica.

\section{Materiales y métodos}

Para la caracterización morfoanatómica del indumento de las especies estudiadas se utilizaron ejemplares procedentes de diferentes herbarios. Se consideraron 5 muestras por especie, a excepción de Nasa perijensis y $N$. venezuelensis para las que se estudiaron 4 (Cuadro 1); con una navaja se realizó un raspado de la superficie (adaxial y abaxial, en caso de estructuras laminares) de cada una de las estructuras vegetativas (tallos y hojas) y reproductivas (lóbulos del cáliz, pétalos, estambres, estaminodios y ovario). Con estas estructuras se prepararon láminas semipermanentes, para lo cual se colocaron en un portaobjeto con solución agua-glicerina (1:1). Con el fin de observar si presentaban algún contenido, no se tiñeron. Para observar las bases y la orientación de los tricomas, las muestras herborizadas se hidrataron y ablandaron, colocando las partes vegetales de interés en etanol al $96 \%$ en baño María a ebullición suave durante 10 minutos; posteriormente se dejaron en lactofenol durante 24 horas, según el procedimiento propuesto por Peña y Saralegui (1982). Las porciones vegetales fueron seccionadas a mano alzada, se tiñeron con azul de toluidina acuosa $(0,5 \%)$, se montaron en agua-glicerina $(\mathrm{V}: \mathrm{V})$ y se sellaron con esmalte transparente para uñas, en el Laboratorio de Anatomía "Antonio Fernández" de la Facultad de Agronomía, Universidad Central de Venezuela (UCV).

Las láminas preparadas se observaron y fotografiaron con un microscopio óptico binocular Leitz-HMLUX 
Cuadro 1. Material de Loasaceae examinado para el estudio del indumento

\begin{tabular}{|c|c|}
\hline Especie & Material* \\
\hline Gronovia scandens & T. Ruiz et al. 5016 (MY), G. Morillo 7616 (MY), B. Trujillo y A. Fernández 273 (MY), E. \\
\hline Klaprothia fasciculata & $\begin{array}{l}\text { Noguera y D. Diamont } 399 \text { (MY), J. Molina s/n. } \\
\text { Aristeguieta } 5388 \text { (VEN), L. Ruiz-Terán y J.A. Dugarte } 15522 \text { (MERF), V. M. Badillo } 4482\end{array}$ \\
\hline K. mentzelioides & $\begin{array}{l}\text { (MY), C. E. Chardon } 199 \text { (VEN), S. S. Tillett 9410-26 (MYF). } \\
\text { J. Steyermark y R. Liesner } 121924 \text { (VEN), J. Steyermark y R. Liesner } 118456 \text { (VEN), G. }\end{array}$ \\
\hline & $\begin{array}{l}\text { Morillo et B. Manara } 1592 \text { (VEN), J. Steyermark et G.C.K. y E. Dunsterville } 100562 \text { (VEN), } \\
\text { Aristeguieta } 740 \text { (VEN). }\end{array}$ \\
\hline Nasa lindeniana & $\begin{array}{l}\text { E. Noguera y P. Villa } 413 \text { (MY), E. Hidalgo y P. Villa } 12 \text { (MY), L. E. Ruiz-Terán } 3192 \\
\text { (MER), M. Weigend } 3601 \text { (MERC), S.S. Renner } 2061 \text { (VEN) }\end{array}$ \\
\hline N. perijensis & S. S. Tillett 747-1043 (MYF), J. Cuatrecasas y R. Romero-Castaneda 25227 (COL), S.S. \\
\hline N. triphylla subsp. papaverifolia & $\begin{array}{l}\text { Tillett et K.W. Hönig } 747 \text { - } 947 \text { (MYF), S. S. Tillett 747-1159 (MYF). } \\
\text { C. E. Benitez } 1757 \text { (MY), S. F. Smith V3151 (VEN), J. Casadiego } 223 \text { (UCOB), N. Xena } 623 \\
\text { (MY), A. Fernández } 3680 \text { (MY). }\end{array}$ \\
\hline N. venezuelensis & $\begin{array}{l}\text { M. Weigend } 3587 \text { (MERC), M. Weigend } 3604 \text { (MERC), Steyermark } 55528 \text { (F), Steyermark } \\
56594 \text { (F). }\end{array}$ \\
\hline Mentzelia aspera & $\begin{array}{l}\text { E. Noguera et al. } 273 \text { (MY), E. Noguera } 396 \text { (MY), E. Noguera y D. Diamont 401(MY), M. } \\
\text { Zabalo } 38 \text { (IRBR), Y. Salazar } 187 \text { (IRBR). }\end{array}$ \\
\hline \multirow[t]{2}{*}{ M. scabra subsp. chilensis } & T. Ruiz y equipo de ecología 2999 (MY), J. Steyermark 57055 (VEN), \\
\hline & $\begin{array}{l}\text { L. Ruiz Terán et M. López Figueiras } 9495 \text { (MERF), S. S. Tillett et R. Hóning de T. 939-20 } \\
\text { (MYF), G. Bunting } 2654 \text { (MY). }\end{array}$ \\
\hline
\end{tabular}

*Acrónimos de acuerdo con Holmgrean et al., 1990.

equipado con cámara fotográfica. El largo de los tricomas se midió con un microscopio óptico binocular LeitzMHLUZ calibrado, equipado con un ocular micrométrico; se efectuaron 30 mediciones para cada tipo de tricoma en cada estructura de las especies estudiadas. Se calculó la media y la desviación estándar en cada caso.

Para describir con detalle la ornamentación y forma de los tricomas se hicieron observaciones con un microscopio electrónico de barrido (MEB) Philips XL 20, en el Centro de Microscopía Electrónica de Barrido de la Facultad de Agronomía de la UCV. Se colocaron porciones de las partes vegetales de interés en cinta doble adherente, sobre un portamuestra, luego se metalizaron con una capa de 15$35 \mathrm{~nm}$ de oro-paladio en un metalizador Bal-Tec SCB 050, se observaron y se fotografiaron. En el caso de órganos laminares se observaron las superficies adaxial y abaxial y en el ovario las paredes dorsales de los carpelos. En la caracterización de los tricomas se siguió la terminología propuesta por Payne (1978), con modificaciones para adaptarla a los tricomas observados.

\section{Resultados}

Se observó que el indumento tiene 2 tipos de apéndices epidérmicos: tricomas y emergencias (Cuadro
2). Los primeros se clasificaron en 2 categorías: tricomas eglandulares (unicelulares y multicelulares) y tricomas glandulares (sólo multicelulares). Los tricomas eglandulares presentan 5 tipos y de 2 a 3 subtipos (Cuadro 2) en los diferentes órganos estudiados en cada una de las especies. A continuación se describen e ilustran los tricomas y emergencias y se sintetiza su distribución en cada uno de los órganos estudiados en el Cuadro 3.

\section{Tricomas \\ A. Tricomas eglandulares \\ A.1. Unicelulares}

Tipo 1. Tricomas cónicos. El ápice es agudo, varían en tamaño; de 50-3700 $\mu \mathrm{m}$ de longitud, pueden ser rectos o curvos; en ocasiones se observan paralelos a la superficie y con el ápice orientado hacia la parte superior del órgano donde se encuentran; cuando están en los márgenes, generalmente se curvan (Figs. 1A-C, 2A-D). Cuando tienen longitudes menores (entre 120 - $160 \mu \mathrm{m}$ ) pueden presentar forma piriforme. Dependiendo de la ornamentación de la pared se distinguen 3 subtipos: 1a) con paredes gruesas, con púas antrorsas o retrorsas, siendo éstas más prominentes en la base; las púas están distribuidas en verticilos a lo largo del eje, la base es angosta (Figs. 1A, 2A); 1b) con las paredes muy delgadas, con púas antrorsas incipientes (Figs. 1B, 2C), su longitud oscila entre $50-420 \mu \mathrm{m}$ de 
Cuadro 2. Clasificación del indumento para Loasaceae de Venezuela

\begin{tabular}{|c|c|c|c|c|}
\hline Indumento & Condición glandular & Condición celular & Tipo & Subtipo \\
\hline \multirow[t]{6}{*}{ I. Tricoma } & A. Eglandular & A.1.Unicelular & 1. Cónico & $1 \mathrm{a}, 1 \mathrm{~b}, 1 \mathrm{c}$ \\
\hline & & & 2. Gloquidiado con ápice uncinado & $2 a, 2 b, 2 c$ \\
\hline & & & 3. Gloquidiado con ápice romo & $3 a, 3 b$ \\
\hline & & & 4. Papila & \\
\hline & & A.2.Multicelular & 5. Dendrítico & \\
\hline & B. Glandular & B.1.Multicelular & & \\
\hline II. Emergencia & & & & \\
\hline
\end{tabular}

Cuadro 3. Distribución del indumento en diferentes órganos de las especies de Loasaceae en Venezuela

\begin{tabular}{|c|c|c|c|c|c|c|c|}
\hline Especie & Tallo & Hojas & Lóbulos del cáliz & Pétalos & Estambres & Estaminodios & Ovario \\
\hline Gronovia scandens & Emergencias & $2 \mathrm{a}, 2 \mathrm{c}$ & $1 \mathrm{a}, 1 \mathrm{c}, 2 \mathrm{a}, 2 \mathrm{~b}, 2 \mathrm{c}$ & $2 \mathrm{c}$ & $2 \mathrm{c}$ & - & $1 \mathrm{a}, 2 \mathrm{a}$ \\
\hline $\begin{array}{l}\text { Klaprothia } \\
\text { fasciculata }\end{array}$ & $\begin{array}{l}\mathrm{Ic}, 2 \mathrm{~b} \\
1 \mathrm{a}, 2 \mathrm{c}\end{array}$ & $1 \mathrm{a}, 1 \mathrm{c}, 2 \mathrm{~b}, 2 \mathrm{c}$ & $1 \mathrm{a}, 2 \mathrm{c}$ & $1 \mathrm{a}$ & 4 & $1 b, 4$ & $\begin{array}{c}1 \mathrm{a}, 2 \mathrm{a} \\
2 \mathrm{c}\end{array}$ \\
\hline K. mentzelioides & $2 \mathrm{c}$ & $1 \mathrm{a}, 2 \mathrm{c}$ & $1 \mathrm{a}$ & $1 \mathrm{a}, 4$ & 4 & $1 b, 4$ & $3 b$ \\
\hline Mentzelia aspera & $3 a$ & $1 \mathrm{a}, 2 \mathrm{c}, 3 \mathrm{a}$ & $3 a$ & $1 b$ & - & - & $1 \mathrm{a}, 3 \mathrm{a}$ \\
\hline $\begin{array}{l}\text { M. scabra subsp. } \\
\text { chilensis }\end{array}$ & $1 \mathrm{a}, 2 \mathrm{~b}$ & $1 \mathrm{a}, 2 \mathrm{~b}$ & $1 \mathrm{a}, 2 \mathrm{c}$ & $1 b$ & - & - & $1 \mathrm{a}, 2 \mathrm{c}$ \\
\hline Nasa lindeniana & $1 c, 2 b, 6$ & $1 \mathrm{a}, 1 \mathrm{c}$ & $1 \mathrm{c}, 6$ & $1 \mathrm{a}, 4$ & - & 4 & $1 \mathrm{a}, 2 \mathrm{~b}, 6$ \\
\hline$N$. perijensis & $1 \mathrm{a}, 1 \mathrm{c}, 2 \mathrm{~b}, 6$ & $1 \mathrm{a}, 1 \mathrm{c}, 2 \mathrm{~b}, 6$ & $1 \mathrm{a}, 1 \mathrm{c}, 6$ & $1 \mathrm{a}, 1 \mathrm{c}, 2 \mathrm{~b}, 4$ & - & 4 & $\begin{array}{c}1 \mathrm{a}, 1 \mathrm{c} \\
2 \mathrm{~b}, 6\end{array}$ \\
\hline $\begin{array}{l}\text { N. triphylla subsp. } \\
\text { papaverifolia }\end{array}$ & $1 \mathrm{c}, 2 \mathrm{~b}$ & $1 \mathrm{a}, 1 \mathrm{c}, 2 \mathrm{~b}, 6$ & 1a, $1 \mathrm{c}, 6$ & $1 \mathrm{a}, 2 \mathrm{~b}, 4,6$ & - & 4 & $\begin{array}{c}1 \mathrm{a}, 1 \mathrm{c} \\
2 \mathrm{~b}, 6\end{array}$ \\
\hline N. venezuelensis & $\begin{array}{c}1 \mathrm{a}, 1 \mathrm{c}, 2 \mathrm{~b} \\
5,6\end{array}$ & $1 \mathrm{a}, 1 \mathrm{c}, 6$ & $1 \mathrm{a}, 1 \mathrm{c}, 6$ & $\begin{array}{c}1 \mathrm{a}, 1 \mathrm{c}, 2 \mathrm{~b} \\
4,6\end{array}$ & - & 4 & $\begin{array}{l}1 \mathrm{a}, 1 \mathrm{c}, \\
2 \mathrm{~b}, 5,6\end{array}$ \\
\hline
\end{tabular}

largo, y 1c) con las paredes muy gruesas, lisas, y la base ancha, erectos (Figs. 1C, 2D, 5A, B), éstos son los tricomas de mayor longitud, oscilan entre 700-3700 $\mu \mathrm{m}$ de largo.

Tipo 2. Tricomas gloquidiados de ápice uncinado. Presentan púas apicales retrorsas de diferentes tamaños, siempre son rectos y con paredes gruesas, la base puede ser angosta a ligeramente ensanchada (Figs. 1D-F, 3A-D). Según la distribución y orientación de las púas a lo largo del eje mayor del tricoma y la forma de la base, se pueden diferenciar 3 subtipos: 2a) presenta un ápice con 4 púas pequeñas retrorsas y a lo largo de su eje tiene púas retrorsas que se distribuyen al azar; su base es angosta y lisa y por encima de ella existe un ensanchamiento, confiriéndole una apariencia piriforme (Figs. 1D y 3A). Su longitud oscila entre 99 - $330 \mu \mathrm{m} ; 2$ ) tiene el ápice con 2-4 púas pequeñas, retrorsas; a lo largo del eje son evidentes púas grandes retrorsas, dispuestas al azar y dispersas, en la base por lo general púas antrorsas. (Figs. 1E, 3B, C). Su longitud oscila entre 95 - $230 \mu \mathrm{m}$. Cuando presentan longitudes cortas la base tiende a ensancharse, y 2c) presenta ápice con 4 púas pequeñas retrorsas; a lo largo del eje posee púas retrorsas, pequeñas, dispuestas aproximadamente en hileras, es de aspecto filiforme y tiene la base ensanchada (Figs. 1F, 3D). Su longitud oscila entre 52 - $245 \mu \mathrm{m}$.

Tipo 3. Tricomas gloquidiados de ápice romo. Presentan 4 o más púas retrorsas apicales y a lo largo del eje del tricoma las púas están dispuestas en hileras; siempre son rectos, con paredes gruesas y las bases ligeramente ensanchadas (Figs. 1G, H, 4A, B). Se distinguen 2 subtipos: $3 a$ ) ápice con 4-5 púas retrorsas grandes, muy bien definidas; por debajo del ápice una porción lisa, presentándose luego varias hileras de púas retrorsas a lo largo del eje del tricoma, la base ligeramente ensanchada (Figs. 1G, 4A). Su longitud oscila entre 120 - $600 \mu \mathrm{m}, \mathrm{y}$ 3b) ápice angosto-cupuliforme, liso, rematando en púas retrorsas; púas bien definidas, dispuestas en hileras a lo largo del eje del tricoma, base ligeramente ensanchada (Figs. 1H, 4B). Su longitud promedio es de $464 \mu \mathrm{m}$. 
Tipo 4. Papilas. Son filiformes con ápice redondeado, paredes lisas y delgadas; presentan diferentes longitudes; estos tricomas no fueron medidos (Figs. 1I, 4C).

\section{A.2. Multicelulares}

Tipo 5: Tricomas dendríticos. Compuestos por 5-7 células de paredes lisas y ligeramente gruesas, dispuestas longitudinalmente, las células apicales son agudas (Figs. 1J, 5D, E). La longitud promedio de estos tricomas es de $170 \mu \mathrm{m}$ y son relativamente escasos.

\section{B. Tricomas glandulares}

\section{B.1. Multicelulares}

Tipo 6. Glandulares. Compuestos por 4-6 células, de paredes muy delgadas y la célula apical globosa (Figs. 1K, 4D, 5C). Su longitud oscila entre 230-1330 $\mu \mathrm{m}$.

\section{Emergencias}

Las emergencias son apéndices unicelulares formados por tejido epidérmico y subepidérmico con paredes gruesas y lisas y ápice de doble gancho (Figs. 1L, 6A, B). Su longitud oscila entre 1-2 mm.

Los tricomas cónicos y los 2 tipos de gloquidiados, con sus diferentes subtipos, se observaron en la mayoría de los órganos estudiados (Cuadro 3). El tricoma 1c es un tricoma urticante, que al contacto con la piel produce irritaciones que pueden perdurar al menos entre $30 \mathrm{y}$ 40 minutos (reacción experimentada en campo y con muestras herborizadas). El tricoma dendrítico (tipo 5) sólo se encontró en el tallo y ovario de $N$. venezuelensis y es escaso. En los tallos de $M$. aspera, frecuentemente se observaron insectos muertos sobre tallo y ramas; la tibia y el tarso de la pata de los insectos quedaban enganchados en las púas de los tricomas gloquidiados con ápice uncinado (Fig. 7). En los diferentes órganos de las especies de $\mathrm{Nasa}$ fue común el tricoma glandular (tipo 6). Las emergencias sólo se encontraron en el tallo de G. scandens.

Las hojas y los ovarios son las estructuras que muestran la mayor diversidad de tricomas (Cuadro 3). En las nervaduras de las hojas, generalmente se apreció un indumento denso; en las mismas algunos de los tricomas tienden a estar paralelos a la superficie, lo contrario de la lámina foliar donde los tricomas están erectos. En ambas superficies de las láminas foliares fue frecuente un patrón de distribución de tricomas grandes intercalados con tricomas pequeños.

En los pétalos de M. aspera y M. scabra subsp. chilensis sólo hay tricomas en el ápice, formándose un mechón de tricomas cónicos del tipo 1b. La densidad de tricomas en el exterior de los pétalos fue relativamente mayor que en el interior.

En general los estambres carecen de tricomas, sólo se observaron papilas pequeñas en las 2 especies de Klaprothia y los tricomas gloquidiados 2c en G. scandens.
En las especies donde se presentan estaminodios, también predominan las papilas.

\section{Discusión}

Los resultados revelan que el tipo de apéndice epidérmico presente, así como su ubicación tienen valor diagnóstico para las especies de Loasaceae de Venezuela; confirmando lo señalado por diversos autores para esta familia (Metcalfe y Chalk, 1950; Davis y Thompson, 1967; Barthlott, 1981; Behnke y Barthlott, 1983; Weigend, 2003, Weigend 2007).

Weigend (2003) consideró que en las Loasaceae los numerosos tipos de tricomas pueden agruparse técnicamente en: escábridos-gloquidiados, tricomas urticantes o setas y tricomas glandulares. En líneas generales, lo observado en nuestro estudio coincide con lo que señala Weigend (2003). Sin embargo, a tales tipos habría que agregar los tricomas dendríticos, que también Davis y Thompson (1967) registraron para Cevallia (Gronovioideae) y fueron observados aquí por primera vez en $N$. venezuelensis (Loasoideae), y las emergencias, presentes en G. scandens (Gronovioideae).

La clasificación propuesta por Weigend (2003) para tricomas es muy amplia, ya que se agrupan tricomas que no son exactamente iguales morfológicamente. En este trabajo se aporta evidencia para reconocer subtipos, los cuales ofrecen información útil para separar los géneros.

Los tricomas de tipo cónico y gloquidiado con ápice uncinado y sus respectivos subtipos son comunes en todas las especies estudiadas; estos tipos coinciden con los tricomas considerados como comunes en Loasaceae por Davis y Thompson (1967) y Poston y Nowicke (1993). Según Poston y Nowicke (1993) en Gronovia hay 2 tipos especializados de tricomas; de los cuales, 1 se asemeja al tricoma cónico subtipo 1c, ya que el tricoma observado en este estudio no presentó un ápice globoso sino agudo. El otro tipo denominado two-hooked trichome, corresponde a una emergencia, según los resultados aquí obtenidos, y se ha considerado como tal debido a la participación de tejido epidérmico y subepidérmico en su estructura. Estas emergencias, al parecer, son exclusivas de la subfamilia Gronovioideae, ya que sólo se encuentran en Gronovia, y según Davis y Thompson (1967) y Poston y Nowicke (1993) en Fuertesia, ambos géneros de hábito trepador.

En Mentzelia, específicamente en $M$. aspera, además de los tipos de tricomas comunes, se encontró el tricoma gloquidiado de ápice romo subtipo $3 \mathrm{a}$, que no fue observado en M. scabra subsp. chilensis, lo que permite diferenciar ambas especies y corroborar lo expuesto por Darlington (1934), quien señaló que en muchos casos los 


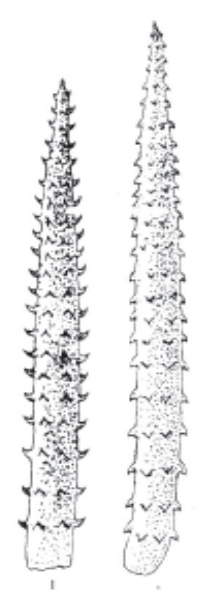

A
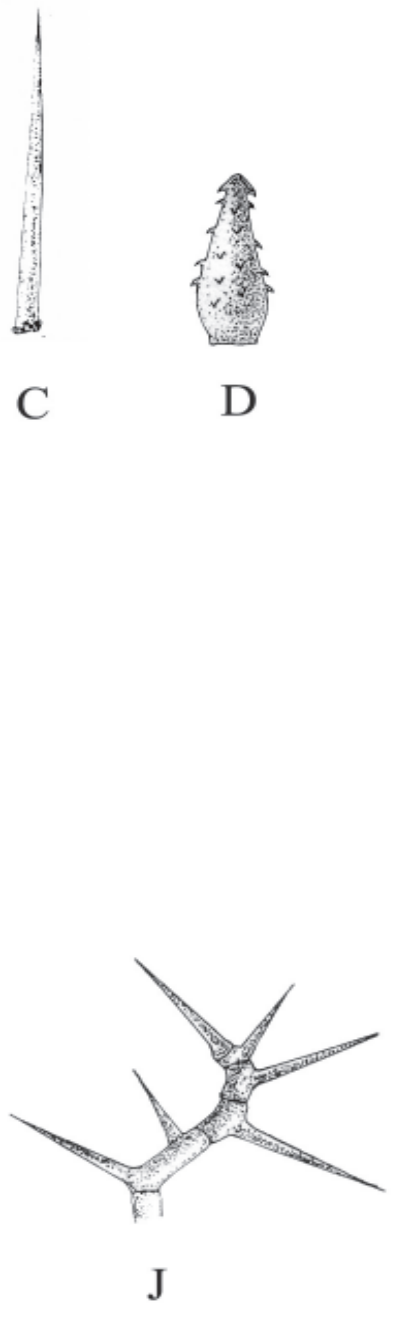

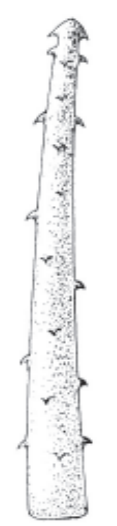

E

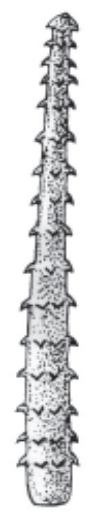

F

B
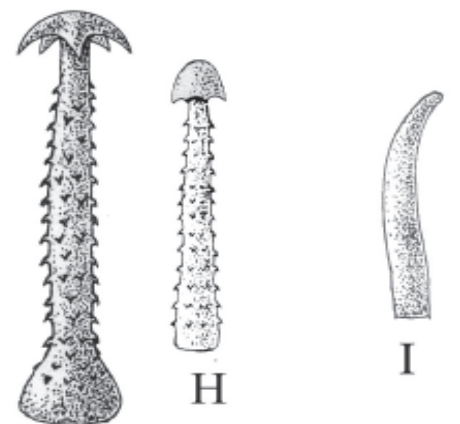

G
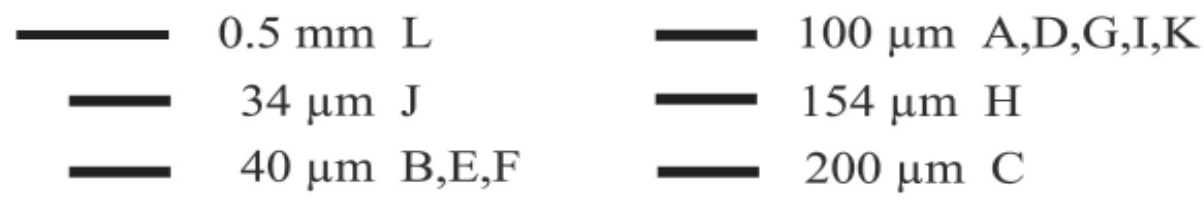

Figura 1. Apéndices epidérmicos en Loasaceae. A-C. Tricomas cónicos: A, subtipo 1a; B, subtipo 1b; C, subtipo 1c. D-F. Tricomas gloquidiados de ápice uncinado: D, subtipo 2a; E, subtipo 2b; F, subtipo 2c. G-H. Tricomas gloquidiados de ápice romo: G, subtipo 3a; H, subtipo 3b. I. Papilas. J. Tricoma dendrítico. K. Tricoma glandular. L. Emergencia. 

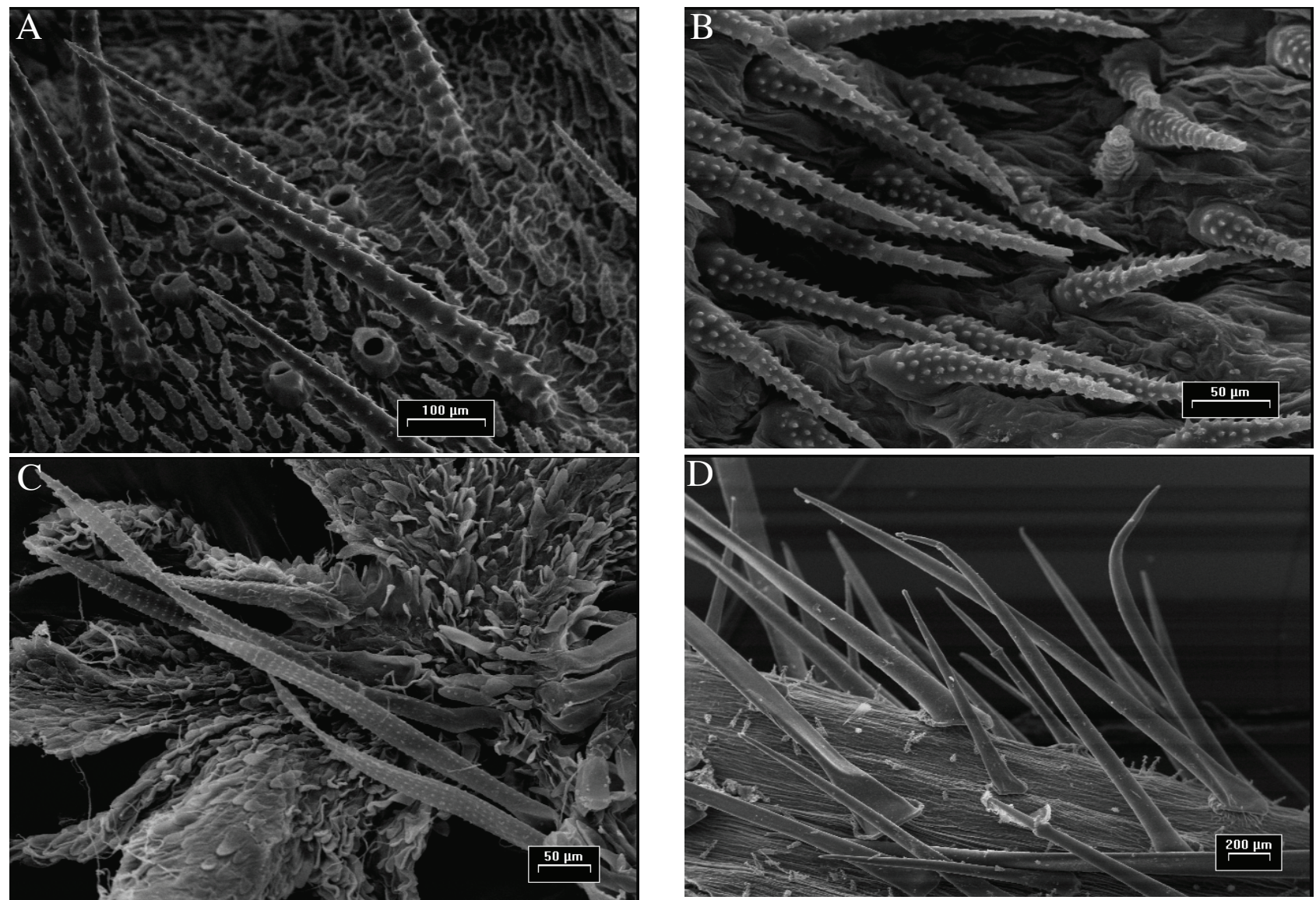

Figura 2. Tricomas cónicos de Loasaceae vistos al microscopio electrónico de barrido. A, subtipo 1a (fruto de G. scandens); B, subtipo 1a (bráctea floral de $K$. fasciculata); C, subtipo 1 b (estaminodio de $K$. mentzelioides); D, subtipo 1c (tallo de $N$. lindeniana).
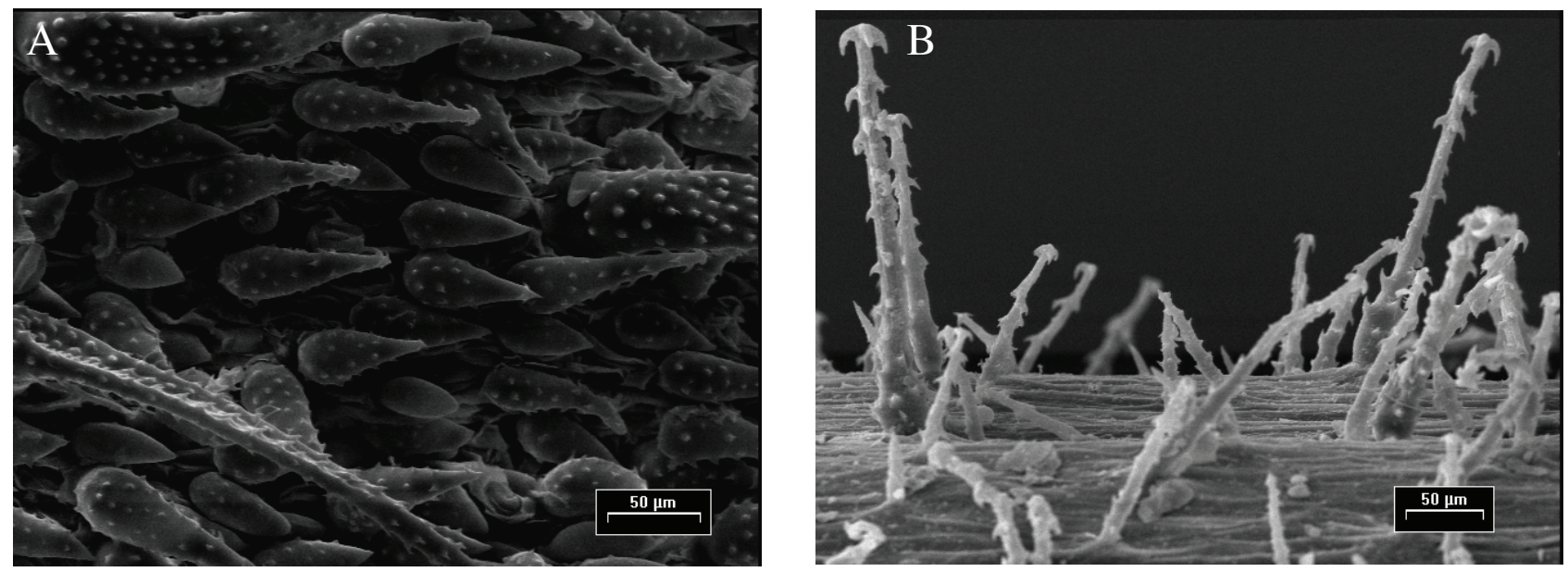

Figura 3. Tricomas gloquidiados de ápice uncinado de Loasaceae vistos al microscopio electrónico de barrido. A, subtipo 2a (fruto de G. scandens); B, subtipo 2b (tallo de M. scabra subsp. chilensis). 

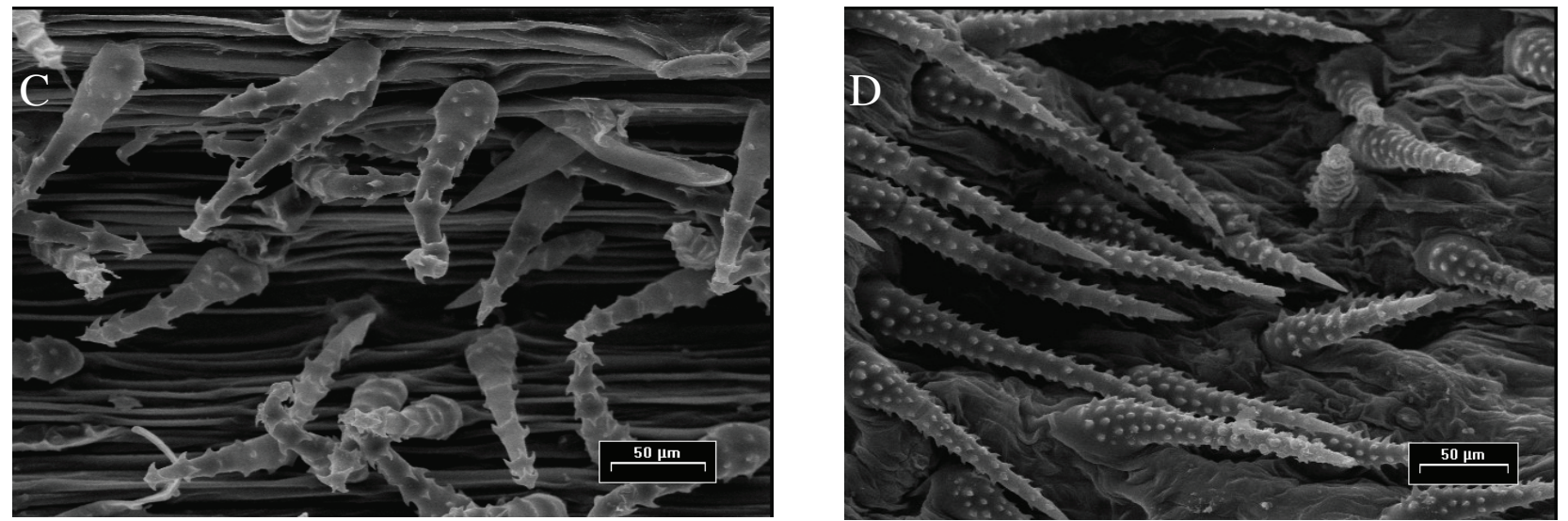

Figura 3. Continuación. Tricomas gloquidiados de ápice uncinado de Loasaceae vistos al microscopio electrónico de barrido. C, subtipo 2b, variación cuando tiene la base ancha (tallo de G. scandens); D, subtipo 2c (ovario de K. fasciculata).
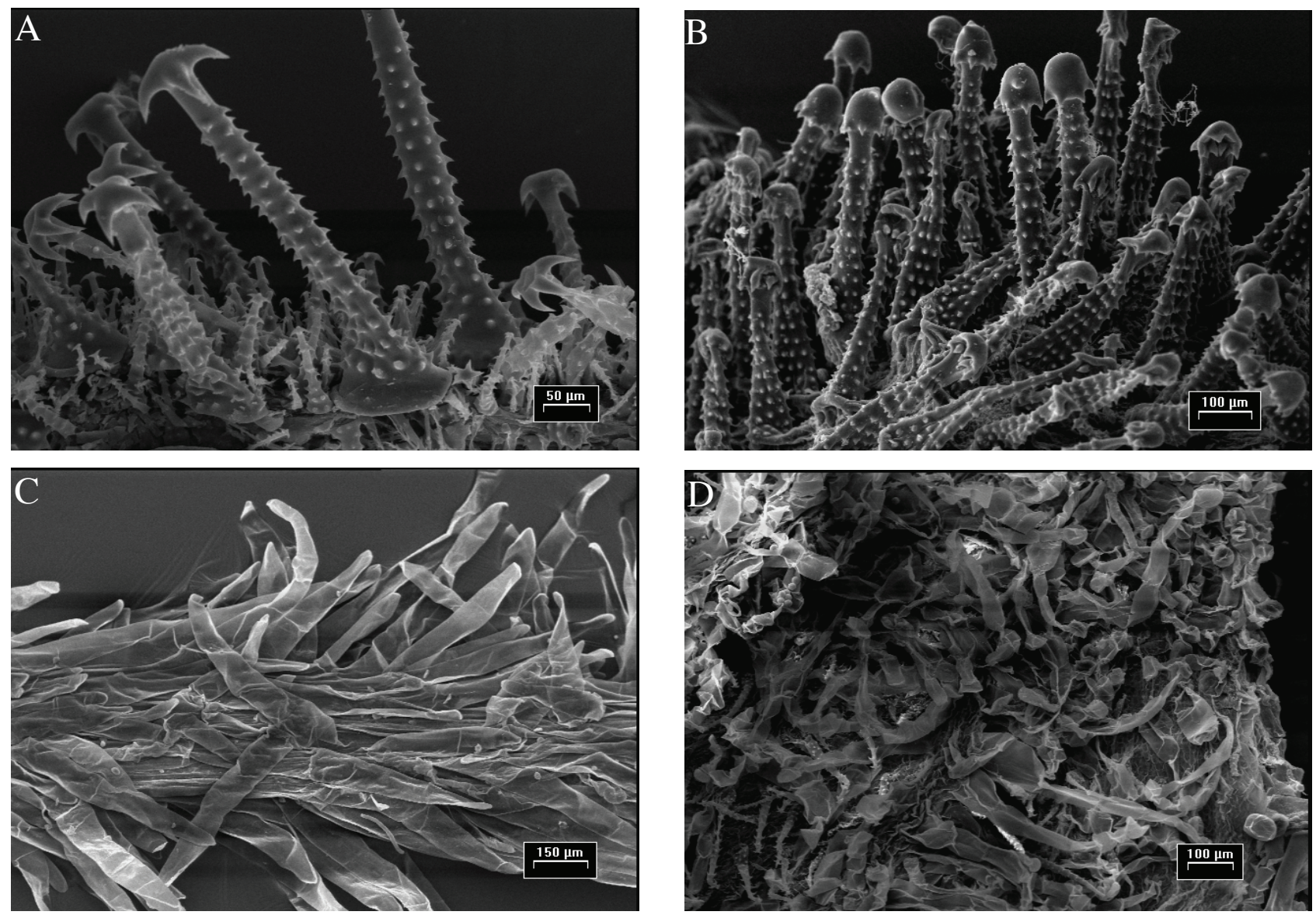

Figura 4. Tricomas gloquidiados de ápice romo, papilas y glandulares de Loasaceae vistos al microscopio electrónico de barrido. A, subtipo 3a (pecíolo de M. aspera); B, subtipo 3b (ovario de K. mentzelioides); C, tipo 4 (estaminodio N. lindeniana); D, tipo 6 (pétalo N. triphylla subsp. papaverifolia). 

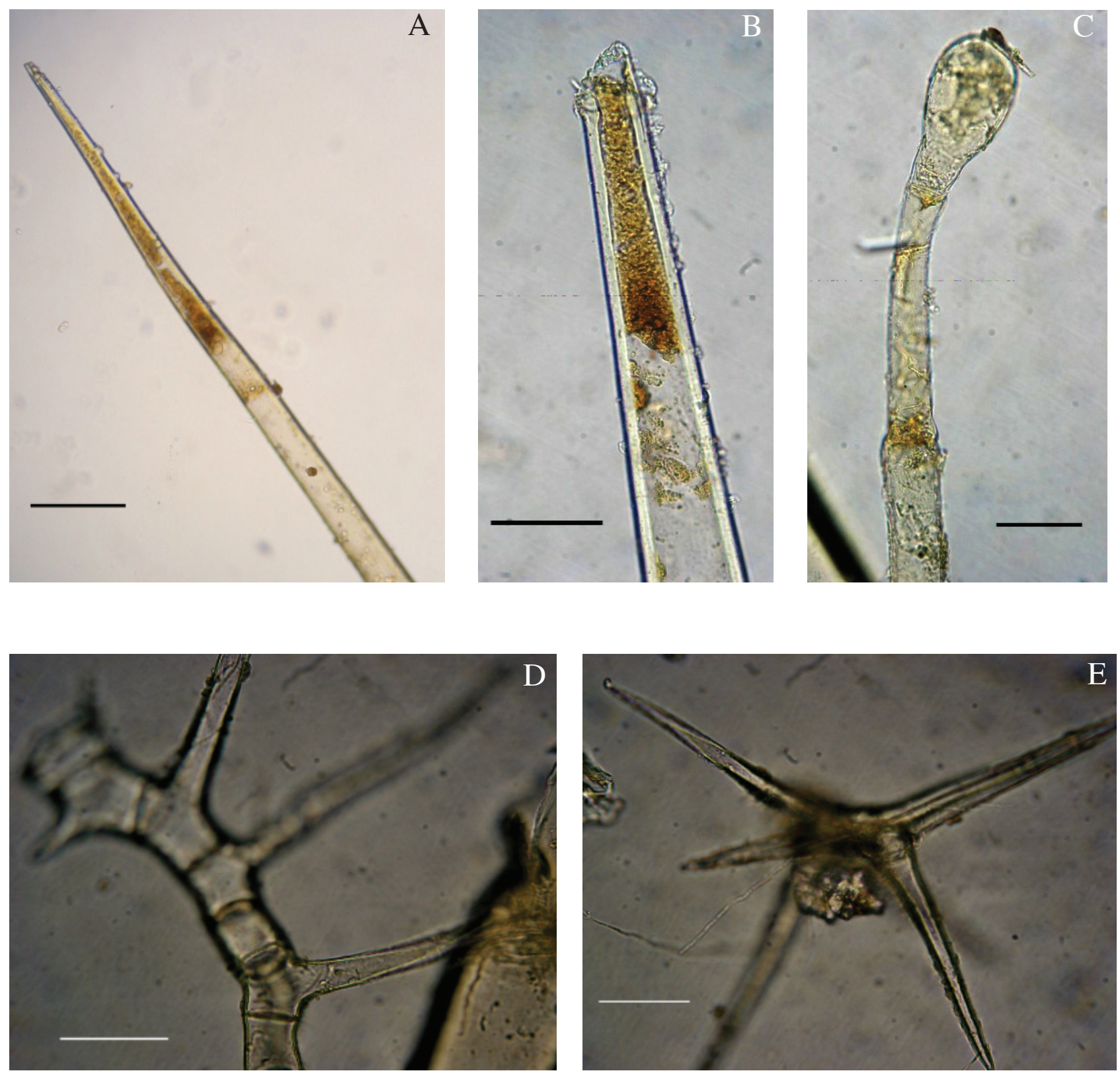

Figura 5. Tricomas de Loasaceae presentes en Venezuela vistos al microscopio óptico. A y B, subtipo 1c (fruto N. venezuelensis), obsérvese la sustancia amarilla en el interior del tricoma; C, tricoma tipo 6 (pétalo de N. venezuelensis); D y E, tricoma tipo 5 (tallo de N. venezuelensis). Barra: A y $\mathrm{B}=100 \mu \mathrm{m}, \mathrm{C}=50 \mu \mathrm{m}, \mathrm{D}, \mathrm{E}=20 \mu \mathrm{m}$. 

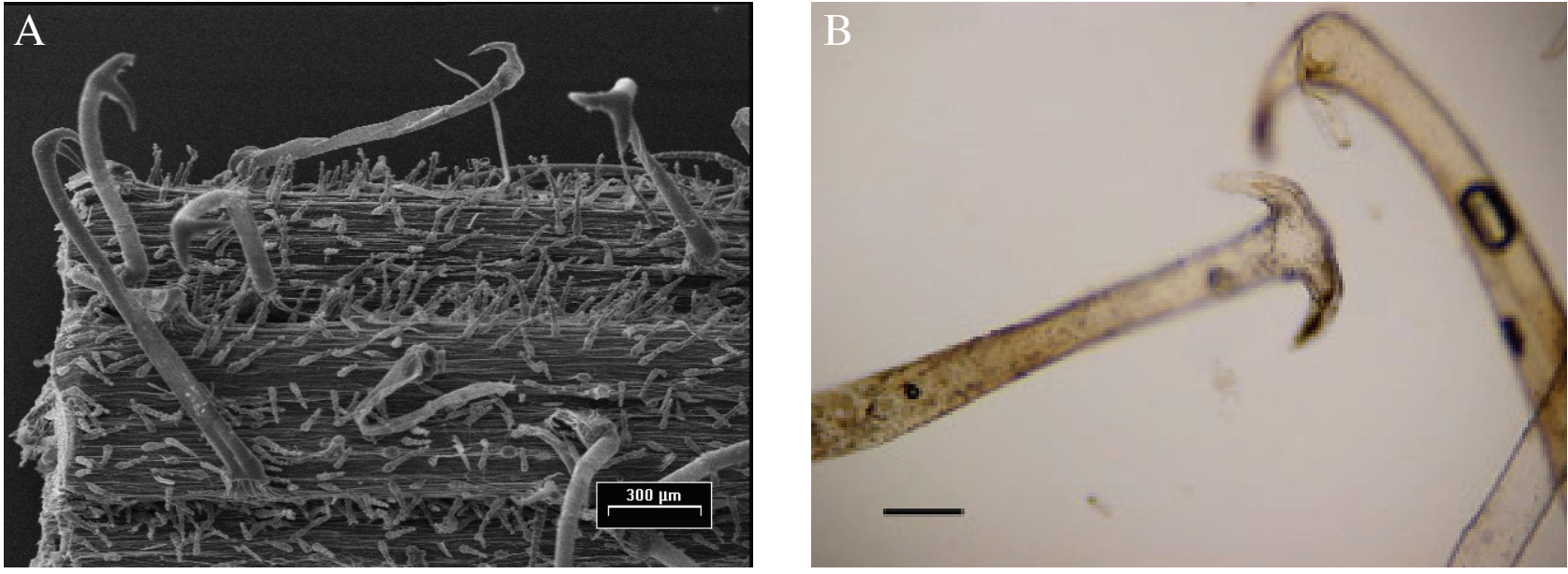

Figura 6. Emergencias del tallo de G. scandens. A, vistas al microscopio electrónico de barrido; B, vistas al microscopio óptico. Barra $=100 \mu \mathrm{m}$.

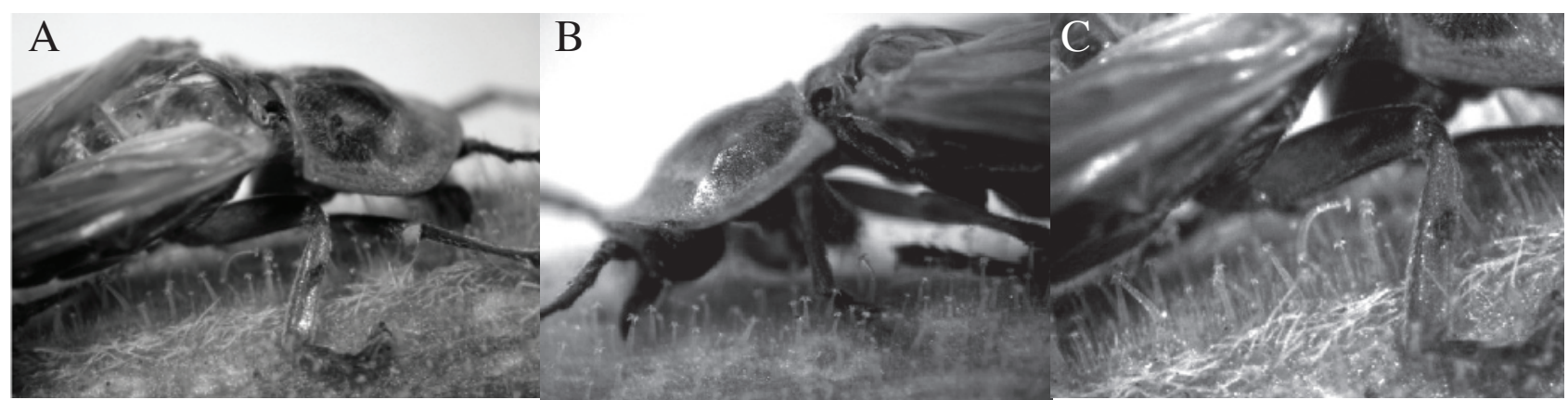

Figura 7. A y B, insecto muerto en tallo de M. aspera; C, detalle de la pata del insecto y tricomas gloquidiados tipo 3a, en los cuales quedan enganchadas las patas de los insectos.

tricomas de Mentzelia proporcionan una herramienta para diferenciación específica. En su trabajo sobre Mentzelia en América del Sur, Weigend (2007) refiere la presencia de tricomas gloquidiados y escábridos (caracterizados por él en 2003) en todos los órganos de las plantas; sin embargo, en su clave para separar las especies de este género sólo utiliza el indumento del ovario, ya que las diferencias son más marcadas en este órgano que en hojas y tallos. Por otro lado, los tricomas subtipos 1a, 2b, 2c registrados en ambas especies de Mentzelia coinciden con los tricomas descritos por Eisner et al. (1998) en las hojas y ramas de M. pumila. Dichos autores consideraron que esos apéndices pueden servir como garfios o podrían funcionar para perforar insectos y restringir su presencia.

En las observaciones que se realizaron en material herborizado y fresco se pudo apreciar la presencia de insectos secos en tallos y ramas, enganchados en estos tricomas; en algunos casos, las púas retrorsas de los tricomas perforan el cuerpo de los insectos o las patas de éstos, quedando enganchadas en las púas e impidiendo al insecto la salida de la planta; de acuerdo con ello se puede considerar que los tricomas sirven como un medio de protección contra depredadores (Raven et al., 1999).

Para las especies de Klaprothia se hallaron diferencias básicamente en el indumento del ovario. Klaprothia fasciculata presenta tricomas subtipos 1a, 2a y 2c, mientras que en $K$. mentzelioides sólo se observaron tricomas subtipo 3b. siendo la única especie con este tipo de tricoma, lo que permite distinguirla del resto de las especies estudiadas. El indumento observado en el ovario de las 2 especies de este género coincide con lo que registran Poston y Nowicke (1993).

Según Dostert y Weigend (1999) en Nasa se han encontrado 3 tipos de tricomas uniseriados: con ápice glandular, escábridos-gloquidiados y urticantes $=$ setas. Los tricomas escábrido-gloquidiados, según Weigend 
(2003), presentan las siguientes características. Escábridos: si los apéndices son más cortos que el diámetro del eje y gloquidiados si son más largos y con forma de ganchos. Los llamados tricomas escábrido-gloquidiados descritos por Dostert y Weigend (1999) coinciden con el tricoma tipo 2 con sus 3 subtipos caracterizados en este estudio. Las observaciones realizadas en Nasa corroboran la presencia de estos tipos de tricomas señalados por Doster y Weigend (1999); sin embargo, también se reconocieron otros tipos de tricomas no señalados en la literatura.

En Nasa se registró la mayor diversidad de tipos de tricomas, encontrándose eglandulares uni- y multicelulares, y glandulares multicelulares. En este trabajo se registra un nuevo tricoma eglandular multicelular, el dendrítico, que se observó exclusivamente en el tallo, las hojas, los lóbulos del cáliz y el ovario de $N$. venezuelensis. Es de destacar que en el género Nasa la presencia de papilas es un rasgo consistente y muy evidente en los estaminodios y estambres de todas las especies estudiadas. Otro tricoma frecuente y sólo observado en Nasa es el tricoma glandular tipo 6.

Es importante señalar que en todas las especies se observó que los tricomas presentaban en su interior un contenido de color amarillo visto al microscopio de luz, como lo describen Metcalfe y Chalk (1950), quienes consideran que se trata de una sustancia irritante que es liberada por los tricomas causando daños en la piel de animales. En el nivel de subfamilia, tanto en Gronovioideae como en Mentzelioideae, sólo se observaron tricomas eglandulares unicelulares, a diferencia de Loasoideae donde se encontraron tricomas eglandulares uni- y multicelulares y tricomas glandulares multicelulares (Cuadro 4). Por otra parte, sólo la subfamilia Gronovioideae presentó emergencias. También es importante destacar que únicamente en la subfamilia Loasoideae se registraron tricomas dendríticos.

A continuación se presenta una clave para separar los géneros de Loasaceae presentes en Venezuela, basada en los caracteres de sus apéndices epidérmicos:

1. a) Estaminodios ausentes .......................................... 2

b) Estaminodios presentes ......................................... 3

2. a) Emergencias presentes en tallos y pecíolos. Estambres con tricomas tipo 2c. Pétalos sólo con tricomas $2 \mathrm{c}$

Gronovia

b) Emergencias ausentes en tallos y pecíolos. Estambres glabros. Pétalos sólo con tricomas $1 \mathrm{~b}$

Mentzelia

3. a) Estaminodios con tricomas 1 b y 4 . Estambres sólo con tricomas tipo 4. Tricomas multicelulares glandulares ausentes

Klaprothia

b) Estaminodios sólo con tricomas tipo 4. Estambres glabros. Tricomas multicelulares glandulares presentes .....
Cuadro 4. Tipos de tricomas y emergencias presentes en subfamilias de Loasaceae en Venezuela

\begin{tabular}{lcl}
\hline Subfamilia & Tipos de tricomas & Emergencias \\
\hline Gronovioideae & 1a, c, 2a, b, c & Presente \\
Loasoideae & 1a, b, c, 2a, b, c, 3b, 4, 5, 6 & Ausente \\
Mentzelioideae & 1a, b, c, 2b, c, 3a, 4 & Ausente \\
\hline
\end{tabular}

\section{Agradecimientos}

Las autoras desean agradecer a FUNDACITE Aragua por el financiamiento parcial $\mathrm{N}^{\circ} 1695$ para la realización de este estudio. Al personal del Centro de Microscopia de la Facultad de Agronomía de la Universidad Central de Venezuela. Al personal del Laboratorio de Anatomía Vegetal Prof. "Antonio Fernández" de la Facultad de Agronomía de la Universidad Central de Venezuela. Al TSU Edgar Esculpi por la elaboración de la ilustración.

\section{Literatura citada}

Barthlott, W. 1981. Epidermal and seed surface characters of plants: systematic applicability and some evolutionary aspects. Nordic Journal of Botany 1:345-355.

Behnke, H. y W. Barthlott. 1983. New evidence from the ultrastructural and micromorphological fields in angiosperm classification. Nordic Journal of Botany 3:43-66.

Bremer, K., M. Chase y P. Stevens. 1998. An ordinal classification for the families of flowering plants. Annals Missouri Botanical Garden 85:531-564.

Darlington, J. 1934. A monograph of the genus Mentzelia. Annals Missouri Botanical Garden 21:103-226.

Davis, W. y H. Thompson. 1967. A revision of Petalonyx (Loasaceae) with a consideration of affinities in subfamily Gronovioideae. Madroño 19:1-32.

Dostert, N. y M. Weigend. 1999. A synopsis of the Nasa trhiphylla complex (Loasaceae), including some new species and subspecies. Harvard Papers in Botany 4:439-467.

Eisner, T., M. Eisner y E. Hoebeke. 1998. When defense backfires: detrimental effect of a plant protective trichomes on an insect beneficial to the plant. Proceedings of the National Academy of Sciences 95:4410-4414.

Holmgren, P. K., H. N. Holmgren y L. C. Barnett. 1990. Index herbariorum. Part I: The herbaria of the world. New York Botanical Garden, New York. 452 p.

Hufford, L. 1988. Seed morphology of Eucnide and other Loasaceae. Systematic Botany 13:154-167.

Hufford, L. 1989. Structure of the inflorescence and flower of Petalonyx Linearis (Loasaceae). Plant Systematic Evolution 163:211-226.

Hufford, L., M. Mcmahon, A. Sherwood, G.Reeves y M. Chase. 2003. The major clades of Loasaceae: phylogenetic analysis 
using the plastid matk and trnl-trnf regions. American Journal of Botany 90:1215-1228.

Judd, S. W., C. Campbell, E. Kellogg, P. Stevens y M. J. Donoghue. 2008. Plant systematics. A phylogenteic approach. Sinauer, Sunderland, Massachusetts. $611 \mathrm{p}$.

Lawrence, G. 1989. Taxonomy of vascular plants. Macmillan, New York. 823 p.

Moody, M. y L. Hufford. 2000. Floral ontogeny and morphology of Cevallia, Fuertesia, and Gronovia (Loasaceae subfamily Gronovioideae). International Journal of Plant Sciences 161:869-883.

Moody, M., L. Hufford, D. Soltis y P. Soltis. 2001. Phylogenetic relationships of Loasaceae subfamily Gronovioideae inferred from matk and its sequence data. American Journal of Botany 88:326-336.

Metcalfe, C. y L. Chalk. 1950. Anatomy of the dicotyledons. Leaves, stem and wood in relation to taxonomy with notes on economic uses. I. Clarendon, Oxford. 724 p.

Noguera, E. 2006. Estudio taxonómico, palinológico y micromorfológico del indumento y la cubierta seminal de Loasaceae Juss. en Venezuela. Tesis maestría, Facultad de Agronomía, Universidad Central de Venezuela, Maracay. $145 \mathrm{p}$.

Payne, W. 1978. A glossary of plant hair terminology. Brittonia 30:239-255.

Peña, E. y H. Saralegui. 1982. Técnicas de anatomía vegetal. La
Habana. 100 p.

Poston, M. y J. Nowicke. 1993. Pollen morphology, trichome types, and relationships of the Gronovioideae (Loasaceae). American Journal of Botany 80:689-704.

Raven, P., R. Evert y S. Eichhorn. 1999. Biology of plants, sixth edition. Freeman, New York. 944 p.

Theobald, W., J. Krahulik y R. Rollins. 1979. Trichome description and classification. In Anatomy of the dicotyledons, vol. I, C. Metcalfe y L. Chalk (eds.). Clarendon, Oxford. p. 668-669.

Thompson, H. y W. Ernst. 1967. Floral biology and systematic of Eucnide (Loasaceae). Journal of the Arnold Arboretum 48:56-88.

Weigend, M. 1997. Nasa and the conquest of South America. $\mathrm{Ph}$. D. Thesis, Ludwig-Maximillians Universität, Munich. $249 \mathrm{p}$.

Weigend, M. 2001. Loasaceae. In Flora de Colombia, R. Bernal y E. Forero (eds.). Monografía 22. Universidad Nacional de Colombia, Bogotá. 100 p.

Weigend, M. 2003. Loasaceae. In The families and genera of vascular plants.VI. Flowering plants-Dicotyledons, Celastrales, Oxalidales, Rosales, Cornales, Ericales. K. Kubizki (ed.). Springer, Berlin. 489 p.

Weigend, M. 2007. Systematics of the genus Mentzelia (Loasaceae) in South America. Annals of the Missouri Botanical Garden 94:655-689. 